\title{
Comparações entre os índices de qualidade de vida em mulheres com incontinência urinária submetidas ou não ao tratamento cirúrgico
}

\author{
Comparisons of quality life index between women with urinary incontinence submitted or not to \\ surgical treatment
}

Antonio Pedro Auge ${ }^{1}$, Carla Monteiro Zucchi², Fernanda Moretzsohn Portella da Costa ${ }^{2}$, Karina Nunes ${ }^{2}$, Livia Porto de Medeiros Cunha ${ }^{2}$, Paula Voltarelli Franco da Silva ${ }^{2}$, Tatiana Umeta Ramos ${ }^{2}$

\section{RESUMO}

Objetivo: analisar o impacto da incontinência urinária sobre a qualidade de vida de mulheres submetidas ou não ao tratamento cirúrgico. Métodos: sessenta mulheres com incontinência urinária de esforço foram entrevistadas e divididas em dois grupos classificados como: grupo S-CIR, incluindo 30 mulheres ainda não submetidas ao tratamento cirúrgico para incontinência urinária e grupo C-CIR, incluindo 30 mulheres já submetidas à cirurgia. Os escores obtidos após o somatório dos pontos atribuídos a cada questão do questionário foram comparados entre os dois grupos. O número de pacientes que mostraram comprometimento na qualidade de vida devido a sintomas específicos da incontinência foi também comparado. Os resultados foram analisados pelo teste de variância e pelo teste $\chi^{2}$, quando aplicáveis. Resultados: sintomas, limitações e preocupações relacionadas à incontinência urinária exerceram impacto negativo na qualidade de vida das pacientes do grupo S-CIR. O comprometimento durante exercícios físicos, atividades domésticas e atividades diárias no trabalho foi o aspecto afetado mais relevante nas pacientes do grupo S-CIR, assim como também relataram mais cansaço, constrangimento e nervosismo em demasia. Perda de urina durante o esforço, seguida por forte desejo de urinar, foram também aspectos significativamente relevantes quando os grupos foram comparados. Conclusões: o estudo permitiu a identificação e a quantificação dos transtornos na qualidade de vida decorrentes da incontinência urinária e demonstrou que estes transtornos tornam-se menos importantes ou até mesmo desaparecem em mulheres submetidas à cirurgia.

PALAVRAS-CHAVE: Incontinência urinária por estresse; Incontinência urinária por estresse/cirurgia; Qualidade de vida

\section{ABSTRACT}

Purpose: to analyze the impact of urinary incontinence on women's quality of life, submitted or not to surgical treatment. Methods: sixty women with urinary incontinence during stress were interviewed and divided into two groups classified as: S-CIR group, including 30 women not yet submitted to specific surgical treatment for urinary incontinence, and C-CIR group, including 30 women who had already undergone surgery. The scores obtained after the addition of values attributed to each question of the questionnaires were compared between the two groups. The number of patients who showed any impairment on quality of life due to specific symptoms of incontinence was also compared between groups. Data were analyzed by the variance test and the $\chi$-square test, when applicable. Results: symptoms, limitations and concerns related to urinary incontinence exhibited a strong negative impact on quality of life in patients from the S-CIR group. Impairment during physical exercises, domestic activities and daily working activities was the most important affected aspect in patients of the S-CIR group. Furtherimore, patients of the S-CIR group reported more fatigue, embarrassment, and excessive nervousness. Urine loss during stress followed by urgency were also significantly relevant aspects when groups were compared. Conclusions: the study allowed the identification and quantification of the derangements of quality of life due to urinary incontinence and demonstrated that these derangements either become less important or even disappear in women submitted to surgery.

KEYWORDS: Urinary Incontinence, stress; Urinary Incontinence, stress/surgery; Quality of life

Trabalho realizado no Departamento de Ginecologia e Obstetrícia da Santa Casa de Misericórdia de São Paulo (SP), Brasil.

1 Professor Assistente e Doutor do Departamento de Obstetrícia e Ginecologia da Santa Casa de Misericórdia de São Paulo (SP), Brasil.

2 Acadêmicas da Faculdade de Ciências Médicas da Santa Casa de Misericórdia de São Paulo (SP), Brasil.

Correspondência: Antonio Pedro Auge

Rua Joaquim Floriano, 72 conj. 88 - Itaim Bibi - 04534-000 - São Paulo - SP - Tel. (11) 3168-4848 - Fax (11) 3078-7733 - e-mail: pedroauge@terra.com.br

Recebido em: 28/11/2005 Aceito com modificações em: 12/7/2006

Rev Bras Ginecol Obstet. 2006; 28(6): 352-7. 


\section{Introdução}

A incontinência urinária (IU), segundo o Comitê de Padronização da Sociedade Internacional de Continência ${ }^{1}$, é definida como qualquer perda involuntária de urina. Afeta aproximadamente $18 \%$ das mulheres com mais de 30 anos de idade ${ }^{2}$, embora seja afecção subdiagnosticada, uma vez que parte das mulheres não relata a perda de urina, por constrangimento ou até por considerarem-na um processo natural.

A incontinência urinária de esforço (IUE) é definida como toda perda de urina durante esforço ou exercício físico, espirro ou tosse ${ }^{1}$. A IUE é confirmada durante o estudo urodinâmico, pela perda de urina através do meato externo da uretra quando a pressão intravesical excede a pressão máxima de fechamento uretral, na ausência de contração do músculo detrusor (definição urodinâmica) ${ }^{3}$. Esse quadro tende a ser progressivo, com perdas periódicas decorrentes de esforços cada vez menores. A perda de qualquer função fisiológica - como a capacidade de repleção e esvaziamento vesical adequado -, mesmo que temporária, é limitante e afeta aspectos sociais e psicológicos, com importante comprometimento na qualidade de vida $(\mathrm{QV})$ da paciente.

O termo QV tem enfoque multidimensional e seu conceito é subjetivo. Apesar de possuir muitas definições, é sabido que tem o intuito de avaliar o efeito de determinados acontecimentos e aquisições na vida das pessoas. Entre eles, estão incluídas as condições de saúde física, funções cognitivas, a satisfação sexual, as atividades do cotidiano, o bem-estar emocional e a vida familiar e social. Quando esses acontecimentos estão ligados à saúde, baseiam-se em dados mais objetivos e mensuráveis e dizem respeito ao grau de limitação e desconforto que a doença ou sua terapêutica acarretam ao paciente e à sua vida ${ }^{4}$, enfim, o quanto o adoecer altera a QV.

A QV das mulheres incontinentes é afetada de diversas maneiras. Passam a se preocupar com a disponibilidade de banheiros, envergonham-se com o odor de urina e sentem-se freqüentemente sujas, chegando a apresentar lesões cutâneas como dermatite amoniacal e infecções urinárias repetidas. Muitas apresentam dificuldade no intercurso sexual, seja por perda de urina, pelo medo de interrompê-lo para urinar ou simplesmente por vergonha perante o parceiro. Alguns sintomas associados à IU afetam a qualidade do sono das mulheres, como a noctúria e a enurese noturna. A privação de sono pode levar ao cansaço e à diminuição da energia.

Geralmente, as pacientes com IU apresentam idade mais avançada, por vezes durante o climatério. Evidências sugerem que tais alterações fisiológicas parecem desencadear quadros de depressão e ansiedade, intensificados pelas sensações de baixa auto-estima e insatisfação sexual geradas pela doença, culminando com a auto-exclusão do convívio social. O incômodo causado aos familiares faz da IU a principal causa de internação asilar ${ }^{5}$.

Inúmeros tratamentos clínicos e cirúrgicos foram desenvolvidos desde o final do século 19 e início do século 20, mas os grandes avanços do estudo neurofisiológico da IU e a recuperação da continência datam de alguns anos atrás, a partir dos quais o impacto da IU sobre a qualidade de vida mereceu significância. O estudo funcional como método orientador da terapêutica cirúrgica é inquestionável. Embora faixas sintéticas suburetrais apliquem-se como tratamento dos casos de hipermobilidade da uretra, assim como nos defeitos esfincterianos intrínsecos, ainda há espaço para as colpo-suspensões retropúbicas, desde que bem avaliadas pelo diagnóstico urodinâmico. Apesar desses avanços, os estudos do impacto sobre a qualidade de vida das pacientes portadoras de IU e daquelas submetidas à correção cirúrgica ainda são escassos na literatura científica.

Diante disso, o presente estudo objetivou analisar o impacto da IUE na QV de mulheres tratadas cirurgicamente e naquelas sem tratamento cirúrgico.

\section{Métodos}

Este estudo foi analisado e aprovado pelo Comitê de Ética em Pesquisa da Santa Casa de Misericórdia de São Paulo. Pacientes atendidas no Ambulatório de Uroginecologia do Departamento de Obstetrícia e Ginecologia do Hospital Central da Irmandade da Santa Casa de Misericórdia de São Paulo, no período de maio e junho de 2005, foram avaliadas.

Consideraram-se para a inclusão neste estudo mulheres com diagnóstico de IUE, após avaliação urodinâmica, tendo realizado ou não algum tratamento cirúrgico. Foram excluídas aquelas com incapacidade de comunicação e/ou dificuldade para responder ao questionário e aquelas que, durante a entrevista, apresentaram queixa exclusiva de urge-incontinência.

No total, 60 pacientes foram divididas em dois grupos: 30 pacientes com IUE não submetidas a qualquer tratamento cirúrgico (S-CIR) e 30 pacientes já submetidas à intervenção cirúrgica para correção de IUE durante os últimos 12 meses (C-CIR).

As participantes do estudo foram informadas sobre os objetivos da pesquisa e somente incluídas neste estudo mediante assinatura de termo de consentimento livre e esclarecido. Foi mantido sigilo sobre a identidade das pacientes. Foi empregado o instrumento de entrevista adaptado do questioná- 
rio de QV King's Health Questionnaire ${ }^{6}$, validado por Fonseca et al. (2005) ${ }^{7}$ (Quadro 1).

A aplicação do questionário foi padronizada entre seis entrevistadores sem nenhum tipo de estimulo ou influência nas respostas. A QV das pacientes deste estudo foi avaliada com base em dez itens: avaliação da saúde, IUE como doença, limitação no desempenho de atividades, relações pessoais, preocupações, limitações, psiquismo, sono, energia e sintomas (Quadro 1). Para a análise dos dados colhidos, escala numérica crescente foi atribuída a cada questão referindo, de maneira diretamente proporcional, a intensidade da queixa $(0=$ não/não sei; $1=$ pouco $/$ raramente, $2=$ médio/às vezes, $3=$ muito $/$ sempre) .

Quadro 1 - Dez principais itens avaliados nas pacientes com incontinência urinárias submetidas ou não à cirurgia (questionário de qualidade de vida - King's Health Questionnaire validado por Fonseca et al., 2005) ${ }^{7}$

1. Avaliação da saúde
2. Incontinência urinária como doença
3. Limitação no desempenho de atividades
4. Relações pessoais
5. Preocupações
6. Limitações
7. Psiquismo
8. Sono
9. Energia
10. Sintomas
Pontuação final:
0 = não sei/não; $1=$ pouco/raramente; 2 = médio/às vezes;
3 = muito/sempre
total =
(máx: 90 pontos)

O somatório variou entre 0-90 pontos e foi comparado entre os grupos, por intermédio do aplicativo Epi-Info 6.04B. Os escores de pontos por questão foram analisados pela análise de variância e, as variáveis categóricas, por intermédio do teste de $\chi^{2}$ (teste não-parámetrico). Valores de p inferiores a 0,05 foram considerados estatisticamente significativos.

\section{Resultados}

Não houve diferença significante entre a média ( \pm desvio padrão) das idades da pacientes em cada grupo estudado $(52,3 \pm 12,3$ vs $50,9 \pm 10,2$ anos, respectivamente para $\mathrm{S}-\mathrm{CIR}$ vs C-CIR, $\mathrm{p}=0,642)$.

Quando as médias gerais obtidas nas pacientes dos grupos S-CIR e C-CIR foram comparadas, foi possivel notar que os grupos são divergentes em relação aos aspectos avaliados. A média dos escores obtidos no grupo S-CIR foi 6,3 vezes maior do que aquela obtida no grupo C-CIR $(54,8$ vs 8,8, respectivamente para $\mathrm{S}-\mathrm{CIR}$ vs $\mathrm{C}-\mathrm{CIR}, \mathrm{p}<0,05)$.
Em relação ao comprometimento das atividades diárias, foi possivel notar diferenças entre os grupos S-CIR e C-CIR (4,9 vs 0,4; $\mathrm{p}<0,05)$, assim como em relação às relações pessoais $(3,9$ vs zero; $\mathrm{p}<0,05)$, ao psiquismo $(20,5$ us 4,6; $\mathrm{p}<0,05)$ e à energia da paciente $(6,9$ vs 1,$0 ; p<0,05)$. A IUE causa impacto sobre estes parâmetros quando as pacientes que não reportaram comprometimento da $\mathrm{QV}$ (resposta não) foram comparadas com aquelas cuja resposta referiu qualquer tipo de comprometimento (pouco + médio + muito). A Tabela 1 mostra estes resultados. É possivel notar que nas pacientes em que a cirurgia foi realizada, os aspectos avaliados, na totalidade, parecem comprometer menos de $25 \%$ das pacientes. Por outro lado, em dez dos 14 aspectos investigados, foi possivel observar comprometimento em mais da metade das mulheres do grupo S-CIR. Aspectos como relações familiares, com os amigos e com o parceiro, seguidos do comprometimento das atividades sexuais, parecem ser menos atingidos.

Em análise subseqüente, pacientes que reportaram pouco ou nenhum comprometimento foram comparadas com aquelas que reportaram grande prejuízo na QV (respostas médio + intenso). Dentre os principais fatores complicadores salientam-se os tópicos referentes ao prejuizo durante atividades fisicas $(66,6$ vs 6,6\%), atividades domésticas $(63,3 \%$ vs zero), cansaço excessivo (63,3 vs $3,3 \%)$, constrangimento $(56,6$ vs $3,3 \%)$, nervosismo em demasia (53,3 vs $6,6 \%$ ) e comprometimento das atividades diárias no trabalho (53,3\% vs zero) para os grupos S-CIR e C-CIR, respectivamente, com $\mathrm{p}<0,001$ em todas as comparações.

O questionário abordou também o comprometimento da QV em relação aos sintomas associados à IUE. Em linhas gerais, de acordo com as médias obtidas para o quesito "sintomas", foi possivel notar que a sintomatologia associada à IUE afeta aproximadamente sete vezes mais a QV das pacientes não submetidas à terapêutica cirúrgica (3,0 vs 0,4, respectivamente para S-CIR vs C-CIR, $\mathrm{p}<0,05)$. O mesmo foi observado com preocupações e limitações causadas pela IUE $(15,5$ vs 2,2 , respectivamente para S-CIR vs C-CIR, $\mathrm{p}<0,05)$.

Novamente, foram comparadas as pacientes que reportaram comprometimento da QV (respostas pouco + médio + intenso) com aquelas em cujo questionário não foi reportado nenhum grau de comprometimento (resposta não). Dos sintomas associados à IUE, para as pacientes do grupo sem cirurgia, o que mais incomodou as pacientes foi a perda de urina aos esforços $(93,3 v s 23,3 \%)$ seguido "da forte vontade de urinar" (90,9 vs 30,0\%), do fato de ter que se levantar durante a noite para urinar (83,3 vs 13,3\%), das fortes dores na bexiga $(70,0$ vs $30,0 \%)$ e por molhar a cama durante o 
sono (56,6 vs 3,3\%); respectivamente para S-CIR vs C-CIR, $\mathrm{p}<0,05$ (Tabela 2).

Houve diferença entre os grupos quando as pacientes foram argüidas em relação às suas preocupações e limitações decorrentes da IUE, após a

Tabela 1 - Impacto da incontinência urinária sobre a qualidade de vida em relação às atividades diárias, relações pessoais, psiquismo e energia em 30 pacientes que apresentaram qualquer comprometimento nos tópicos abordados no questionário.

\begin{tabular}{lcc}
\hline Variáveis em porcentagem (\%) & S-CIR & C-CIR \\
\hline Atividades diárias no trabalho & $16(53,3)^{\text {a }}$ & $3(10,0)$ \\
Atividades domésticas & $23(76,6)^{\mathrm{a}}$ & $4(13,3)$ \\
Atividades físicas & $20(66,6)^{\mathrm{a}}$ & $4(13,3)$ \\
Relações com familiares & $11(36,6)^{\mathrm{a}}$ & 0 \\
Relações com amigos & $10(33,3)^{\mathrm{a}}$ & 0 \\
Relações com marido/parceiro & $11(36,6)^{\mathrm{a}}$ & 0 \\
Relações sexuais & $13(43,3)^{\mathrm{a}}$ & 0 \\
Ansiedade & $19(63,3)^{\mathrm{a}}$ & $5(16,6)$ \\
Depressão & $16(53,3)^{\mathrm{a}}$ & $4(13,3)$ \\
Nervosismo & $17(56,6)^{\mathrm{a}}$ & $7(23,3)$ \\
Auto-estima & $15(50,0)^{\mathrm{a}}$ & $3(10,0)$ \\
Constrangimento & $20(66,6)^{\mathrm{a}}$ & $5(16,6)$ \\
Sono comprometido & $19(63,3)^{\mathrm{a}}$ & $6(20,0)$ \\
Cansaço & $21(70,0)^{\mathrm{a}}$ & $4(13,3)$ \\
\hline S-CIR: número de pacientes sem cirurgia. C-CIR : número de pacientes após cirurgia. & &
\end{tabular}

análise das pacientes em relação à resposta [(não) vs (pouco + médio + muito)]. Ter necessidade de trocar a roupa intima todas as vezes que se sentir molhada $(86,6$ vs $30,0 \%)$, localizar banheiros em locais desconhecidos $(86,6$ vs $40,0 \%)$ e ter que usar fraldas ou absorventes $(76,6$ vs $16,6 \%)$ representaram as preocupações mais relevantes no grupo de pacientes que não se submeteram à cirurgia, respectivamente para S-CIR vs C-CIR, $\mathrm{p}<0,05$ (Tabela 2). Mais de $80,0 \%$ das pacientes do grupo S-CIR encaram a IUE como doença. Isto também é demonstrado nas pacientes do grupo C-CIR (53,3\%).

Outras análises foram feitas agrupando-se as pacientes com respostas (não) + (pouco) e comparando-as com pacientes que relataram incômodos relativos à IUE (respostas médio + intenso). Somente $23,3 \%$ das pacientes do grupo S-CIR relataram sentirse incomodadas em relação a seus parentes e amigos por causa do odor da urina. Este perfil não mostrou diferenças quando os grupos foram comparados $(3,3 \%$ no grupo C-CIR, com p=0,052). Limitações ou preocupações referentes ao fato de ter que trocar a roupa intima freqüentemente $(86,6$ vs $13,3 \%$; p<0,05), localização de banheiros em locais desconhecidos $(83,3 \%$ v30,0\%; p<0,05), sentir forte vontade de urinar $(76,6$ vs $10,0 \% ; \mathrm{p}<0,05)$ e a necessidade de utilizar fraldas ou absorventes $(73,3 \%$ x $10,0 \%$; $<<0,05)$ perfizeram os maiores índices de comprometimento no grupo de

Tabela 2 - Impacto da incon tinência urinária sobre a qualidade de vida em relação aos sintomas, preocupações e limitações em 30 pacientes que apresentaram qualquer comprometimento nos tópicos abordados no questionário.

\begin{tabular}{|c|c|c|}
\hline Variáveis em porcentagem (\%) & S-CIR & C-CIR \\
\hline Idas freqüentes ao banheiro & $23(16,6)^{a}$ & $5(16,6)$ \\
\hline Sentir forte vontade de urinar & $27(90,9)^{a}$ & $9(30,0)$ \\
\hline Levantar para urinar à noite & $25(83,3)^{a}$ & $4(13,3)$ \\
\hline Perder urina aos esforços & $28(93,3)^{a}$ & $7(23,3)$ \\
\hline Molhar a cama durante o sono & $17(56,6)^{\mathrm{a}}$ & $1(3,3)$ \\
\hline Perder urina durante a relação sexual & $12(40,0)^{a}$ & $1(3,3)$ \\
\hline Freqüentes infecções urinárias & $11(36,6)$ & $4(13,3)$ \\
\hline Dores na bexiga & $21(70,0)^{a}$ & $9(30,0)$ \\
\hline Utilização de transporte público & $15(50,0)^{a}$ & $1(3,3)$ \\
\hline Empecilho para fazer o que gosta & $21(70,0)^{a}$ & $3(10,0)$ \\
\hline Utilização de fraldas ou absorventes & $23(76,6)^{a}$ & $5(16,6)$ \\
\hline Limitação quanto ao modo de vestir-se & $13(43,3)^{a}$ & $4(13,3)$ \\
\hline Trocar roupa íntima quando fica molhada & $26(86,6)^{a}$ & $9(30,0)$ \\
\hline Ingestão de menores quantidades de líquidos/água & $18(60,0)^{a}$ & $2(6,6)$ \\
\hline Localização do banheiro em lugares novos & $26(86,6)^{a}$ & $12(40,0)$ \\
\hline Mau cheiro quando fica molhada & $22(73,3)^{a}$ & $5(16,6)$ \\
\hline Incômodo causado pelo odor de urina & $8(26,6)^{a}$ & $1(3,3)$ \\
\hline Incômodos físicos causados pela perda de urina & $20(66,6)^{a}$ & $4(13,3)$ \\
\hline Evitar sair de casa devido à perda urinária? & $15(50,0)^{a}$ & $2(6,6)$ \\
\hline Sentir que seu problema é uma doença & $25(83,3)^{a}$ & $16(53,3)$ \\
\hline
\end{tabular}


pacientes não submetidas à cirurgia para correção da IUE, respectivamente para S-CIR vs C-CIR.

\section{Discussão}

O questionário aplicado neste trabalho teve o intuito de demonstrar objetivamente o quanto a IU afeta a QV das mulheres. Em relação às pacientes do S-CIR, pôde-se observar que infecção urinária recorrente foi sintoma considerado pouco significativo na vida dessas mulheres, já que mais da metade das pacientes não o apontaram como queixa importante. Daquelas pacientes que reportaram qualquer grau de incômodo, $70 \%$ apresentou queixa de dor na bexiga, com $46,6 \%$ apresentando o sintoma de modo intenso.

Nosso estudo confirma que a IUE, associada à noctúria, leva a diminuição na disposição física para a realização de atividades e da qualidade do sono das pacientes, pois mais que metade das pacientes do S-CIR relataram que o fato de acordar à noite para urinar afeta de algum modo sua vida por dormirem menos do que gostariam. Foi observado que pelo menos $63,3 \%$ das pacientes se sentem cansadas por não terem um bom sono.

Quanto aos sintomas mais comuns, a IUE foi a segunda questão de maior pontuação (75 pontos). Do total de pacientes analisadas, 96,7\% apresentaram algum comprometimento, sendo em $70 \%$ dos casos de forma intensa.

Quanto às atividades diárias, os resultados comprovam que a IUE leva a prejuizos no trabalho, nas atividades de casa e nas atividades fisicas. A maior parte das entrevistadas mostrou redução da QV nesse aspecto, visto que a questão a respeito das limitações às atividades físicas teve 56 pontos, a questão sobre as limitações nas atividades de casa teve 50 pontos e a questão sobre os limites que a perda urinária impõe sobre o trabalho das mulheres teve 43 pontos.

Nossos resultados demonstram que a IU afeta as relações familiares, amizades, parceiro e as relações sexuais, com a pontuação por questão respectivamente 23, 22, 29 e 45. Quase metade (46\%) das pacientes que freqüentam clínicas uroginecológicas têm freqüência de intercurso sexual diminuída, devido a sintomas como dispareunia, molhar a cama durante a noite ou durante o coito, depressão e dificuldade em atingir o orgasmo. A prevalência da incontinência urinária na s relações sexuais é cerca de $2 \%$ na população ${ }^{8,9}$. Estudo dinamarquês demonstrou que metade das pacientes sexualmente ativas apresentou incontinência urinária durante o intercurso sexual ${ }^{10}$.

Existem muitas razões pelas quais os sintomas da IU afetam a vida sexual, talvez não diretamente relacionadas à perda de urina. A IU pode afetar muitos aspectos da sexualidade, como roupas e aparência ${ }^{11}$. A mulher pode não se sentir atraente, principalmente quando faz uso de absorventes na cama $^{8}$. Há relatos de pacientes que diziam se sentir sujas e mal cheirosas, evitavam a relação sexual ou mesmo a interrompiam para ir ao banheiro ${ }^{12}$. A insatisfação sexual da mulher traduz uma piora na QV individual e conjugal. Estudos têm apontado para estes questionamentos com resultados controversos em relação a seqüelas cirúrgicas, piora e/ou surgimento de dispareunia, alterações vasculares e neurológicas das paredes vaginais, piora da lubrificação e da excitação sexual ${ }^{13}$.

Quanto às preocupações e limitações, número expressivo de entrevistadas mostrou detrimento na $Q V$, especialmente por terem que se trocar quando se molham, por preocuparem-se em saber onde é o banheiro quando vão a lugares novos, por terem que usar fraldas ou absorventes e por se preocuparem com o mau cheiro que a perda involuntária de urina provoca.

Nossos resultados confirmam os da literatura. As necessidades do uso de absorventes e da troca da roupa intima afetam a $\mathrm{QV}^{14}$. O fato de mais de $70 \%$ das entrevistadas sofrerem por constrangimento mostra que a IU leva a prejuízos psíquicos importantes. Quanto ao psiquismo, metade das pacientes apresentou queixa de depressão, resultado três vezes maior do que o publicado por Melville et al. ${ }^{15}$. Nesse estudo a prevalência de depressão como comorbidade à IU foi de $16 \%$, sendo o diagnóstico baseado nos critérios do DSM-IV ${ }^{16}$, diferentemente de nosso estudo, que se baseou em depoimentos subjetivos. De qualquer forma, a coexistência de sintomas depressivos e IU refletem a influência negativa desta na QV das pacientes que, quando depressivas e incontinentes, apresentam maior probabilidade $(1,45)$ de gravidade do quadro psiquiátrico ${ }^{17}$.

Foi possivel observar diferenças significativas entre os resultados de todas as questões comparadas entre os grupos S-CIR e C-CIR. Embora não tenhamos utilizado recursos laboratoriais e de imagem, concluímos, pela avaliação de valores positivos, que as pacientes do grupo C-CIR apresentam melhor QV. A perda de urina aos esforços esteve entre as cinco principais queixas no S-CIR. Essa queixa não se apresentou entre as principais do C-CIR. Isso pode evidenciar uma "cura clínica" da IU após a intervenção cirúrgica, uma vez que essa queixa é base do quadro clínico dessa doença.

As portadoras de IU, com freqüência, não procuram o profissional de saúde com a queixa principal de perda de urina. Muitas vezes, a consideram uma evolução natural, e até algo esperado para as mulheres mais idosas e que já tiveram filhos. É a severidade da perda que desencadeia o desejo para o tratamento da $\mathrm{IU}^{18}$. Mais da metade 
das pacientes $(53,3 \%)$ do C-CIR e $83,3 \%$ do S-CIR considerou a IU como doença. A média de pontos por grupo de questões foi significativamente menor em pacientes após o tratamento cirúrgico, assim como a média total de pontos por paciente evidenciou a diferença na QV entre as pacientes do grupo S-CIR (54,9 pontos) e C-CIR (8,83 pontos).

Em relação ao aspecto emocional, parte das pacientes entrevistadas na fase pré-operatória mostrou comoção durante a aplicação dos questionários. Notou-se que essa reação advém de modificações em suas vidas, pelas dificuldades trazidas pela doença. Parte delas sentiu-se triste e muitas chegaram a chorar durante a entrevista. Ao contrário destas, a grande maioria das pacientes do grupo C-CIR mostrou-se satisfeita, sentindo-se "curadas".

Pôde-se concluir, tendo em vista os resultados, que as alterações biológicas, detalhadas anteriormente, causam diminuição na QV das mulheres com IUE. A aplicação do questionário foi de grande valor e permitiu que as mudanças na vida dessas mulheres fossem quantificadas e qualificadas, pois, por meio de pontuação, foram avaliadas quais queixas eram positivas e o quanto influenciam na vida das pacientes. Em todas as questões, a média de pontos foi maior em pacientes sem tratamento cirúrgico do que em pacientes pós-cirurgia. Pode-se concluir que há menor prejuízo sobre a QV nas pacientes do grupo C-CIR. Nas pacientes do S-CIR, a quantidade de queixas positivas era maioria, o que permite a comprovação de que há impacto importante.

O objetivo do trabalho, avaliar o impacto na QV das pacientes com perda involuntária de urina, e a aplicação do questionário nos permitiram concluir e afirmar que a IUE não é apenas uma alteração fisiopatológica, mas sim uma síndrome, que tem nas queixas psicológicas e sociais os fatores que tornam a paciente doente.

\section{Referências}

1. Abrams P, Cardozo L, Fall M, Griffiths D, Rosier P, Ulmsten $U$, et al. The standardisation of terminology of lower urinary tract function: report from the Standardisation Sub-committee of the International Continence Society. Neurourol Urodyn. 2002;21(2): 167-78.

2. Hannestad YS, Rortveit G, Sandvik H, Hunskaar $\mathrm{S}$; Norwegian EPINCONT study. Epidemiology of Incontinence in the County of Nord-Trondelag. A community-based epidemiological survey of female urinary incontinence: the Norwegian Epincont study. J Clin Epidemiol. 2000;53(11):1150-7.

3. Nygaard IE, Heit M. Stress urinary incontinence. Obstet Gynecol. 2004;104(3):607-20.

4. Silva MAD. A importância da manutenção da quali- dade de vida. Rev Soc Cardiol Estado de São Paulo. 1996;6(5):657-60.

5. Gill TM, Feinstein AR. A critical appraisal of the qualityof-life measurements. JAMA. 1994;272(8):619-26.

6. Kelleher CJ, Cardozo LD, Khullar V, Salvatore S. A new questionnaire to assess the quality of life of urinary incontinent women. Br J Obstet Gynaecol. 1997;104(12):1374-9.

7. Fonseca ESM, Camargo ALM, Castro RA, Sartori MGF, Fonseca MCM, Lima GR, et al. Validação do questionário de qualidade de vida (King’s Health Questionnaire) em mulheres brasileiras com incontinência urinária. Rev Bras Ginecol Obstet. 2005;27(5):235-42.

8. Shaw C. A systematic review of the literature on the prevalence of sexual impairment in women with urinary incontinence and the prevalence of urinary leakage during sexual activity. Eur Urol. 2002;42(5):432-40.

9. Salonia A, Zanni G, Nappi RE, Briganti A, Dehò F, Fabbri F, et al. Sexual dysfunction is common in women with lower urinary tract symptoms and urinary incontinence: results of a cross-sectional study. Eur Urol. 2004;45(5):642-8.

10. Glavind K, Tetsche MS. Sexual function in women before and after suburethral sling operation for stress urinary incontinence: a retrospective questionnaire study. Acta Obstet Gynecol Scand. 2004;83(10): 965-8.

11.Roe B, May C. Incontinence and sexuality: findings from a qualitative perspective. J Adv Nurs. 1999;30(3):573-9.

12.Norton C. The effects of urinary incontinence in women. Int Rehabil Med. 1982;4(1):9-14.

13. Helström L, Nilsson B. Impact of vaginal surgery on sexuality and quality of life in women with urinary incontinence or genital descensus. Acta Obstet Gynecol Scand. 2005;84(1):79-84.

14.Kulseng-Hanssen S, Borstad E. The development of a questionnaire to measure the severity of symptoms and the quality of life before and surgery for stress incontinence. BJOG. 2003;110(11):983-8.

15.Melville JL, Walker E, Katon W, Lentz G, Miller J, Fenner D. Prevalence of comorbid psychiatric illness and its impact on symptom perception, quality of life, and functional status in women with urinary incontinence. Am J Obstet Gynecol. 2002;187(1):80-7.

16.American Psychiatric Association. Diagnostic and Statistical Manual of Mental Disorders DSM-IV-TR (Text Revision). $4^{\text {th }}$ ed. Washington; 2000.

17.Dugan E, Cohen SJ, Bland DR, Preisser JS, Davis CC, Suggs PK, et al. The association of depressive symptoms and urinary incontinence among older adults. J Am Geriatr Soc. 2000;48(4):413-6.

18.Andersson G, Johansson JE, Sahlberg-Blom E, Pettersson N, Nilsson K. Urinary incontinence - why refraining from treatment? A population based study. Scand J Urol Nephrol. 2005;39(4):301-7. 